\title{
A comparison between the digestibility of hay by donkeys and ponies
}

\author{
JL Tisserand, F Faurie, C Cordelet, H Pignon \\ Laboratoire de Recherches de la Chaire de Zootechnie de l'ENSSAA, \\ 26, bd Dr-Petitjean, 21000 Dijon, France
}

The donkey plays a very important part in the rural domestic economy of the Mediterranean area. In order to get more information on its digestion, we compared the digestibility of 2 cocksfoot alfalfa hays given alone (I: $\mathrm{CF}=32$ and $\mathrm{CP}=20 \% \mathrm{DM}$; II: $\mathrm{CF}=39$ and $\mathrm{CP}=15 \% \mathrm{DM})$ to 3 adult male donkeys $(250 \mathrm{~kg})$ and 3 adult male ponies $(180 \mathrm{~kg})$. After a $15 \mathrm{~d}$ adaptation period during which the animals ate ad libitum, digestibility was measured over a period of $6 \mathrm{~d}$. There was then a new period of adaptation to limited quantities of food (40 $\mathrm{g}$ digestible organic matter ingested (DOMI)/kg W0.75) followed by a second $6 \mathrm{~d}$ period of measurement.

In general, donkeys seem to digest dry matter, organic matter and nitrogen better than ponies (table I). Donkeys' feed refusal contains more cellulose and less nitrogen than ponies' feed refusal. The results seem less variable (with a reduced standard error) for donkeys when they are given good quality hay. These preliminary results, which need to be confirmed, agree . with the observations made by Wolter and Velandia (1970) and by Merritt and Pearson (1989).

Merritt JB, Pearson RA (1989) Voluntary food intake and digestion of hay and straw diets by donkeys and ponies. Proc Nutr Soc in press

Wolter R, Velandia J (1970) Rev Med Vet 146, $141-152$

Table I. Donkey and pony hay digestibility.

\begin{tabular}{|c|c|c|c|c|c|c|}
\hline $\begin{array}{c}\text { Diet } \\
\text { or hay }\end{array}$ & & $\begin{array}{c}\text { Dry } \\
\text { matter }\end{array}$ & $\begin{array}{l}\text { Organic } \\
\text { matter }\end{array}$ & $\begin{array}{l}\text { Crude } \\
\text { fiber }\end{array}$ & $\begin{array}{l}\text { Total } \\
\text { nitrogen }\end{array}$ & $\begin{array}{c}D O M \prime \\
g / \mathrm{kg} L W 0.75\end{array}$ \\
\hline a & $\begin{array}{l}\text { donkeys } \\
\text { ponies }\end{array}$ & $\begin{array}{l}62.6 \pm 1.4 \\
57.7 \pm 2.6\end{array}$ & $\begin{array}{l}64.0 \pm 1.5 \\
58.7 \pm 2.6\end{array}$ & $\begin{array}{l}59.7 \pm 3.1 \\
52.9 \pm 3.9\end{array}$ & $\begin{array}{l}76.5 \pm 3.8 \\
71.9 \pm 2.1\end{array}$ & $\begin{array}{l}27.0 \pm 3.4 \\
26.8 \pm 2.7\end{array}$ \\
\hline b & donkeys & $61.2 \pm 0.3$ & $62.1 \pm 0.2$ & $51.6 \pm 0.3$ & $77.6 \pm 0.1$ & $32.4 \pm 4.4$ \\
\hline & ponies & $57.1 \pm 1.0$ & $58.3 \pm 1.1$ & $51.8 \pm 1.4$ & $72.7 \pm 0.7$ & $26.8 \pm 2.7$ \\
\hline$\| \mathbf{a}$ & donkeys & $52.9 \pm 3.2$ & $54.5 \pm 3.4$ & $42.0 \pm 4.2$ & $69.1 \pm 3.2$ & $43.3 \pm 4.7$ \\
\hline$\|^{\mathbf{b}}$ & donkeys & $52.1 \pm 5.0$ & $53.2 \pm 4.7$ & $41.5 \pm 3.9$ & $\begin{array}{l}65.4 \pm 2.5 \\
72.5 \pm 4.5\end{array}$ & $\begin{array}{l}48.0 \pm 4.3 \\
40.4 \pm 4.6\end{array}$ \\
\hline & ponies & $48.1 \pm 4.0$ & $51.3 \pm 3.9$ & $43.1 \pm 6.1$ & $66.7 \pm 1.6$ & $39.6 \pm 5.1$ \\
\hline
\end{tabular}

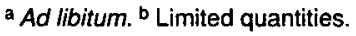

